\title{
Melanoma-Associated Suppression of the Dendritic Cell Lines DC2.4 and Jawsii
}

\author{
Kristian Michael Hargadon, \\ Yonathan Tarekegne Ararso, Osric Anthony Forrest and Chad Mason Harte
}

Department of Biology, Hargadon Laboratory, Hampden-Sydney College, Hampden-Sydney, VA, USA

Received 2012-06-19, Revised 2012-10-17; Accepted 2012-11-3

\begin{abstract}
Dendritic cells function as potent regulators of both innate and adaptive immunity to tumors and the regulatory activities of these cells are tightly linked to their maturation and activation status. Despite the critical role played by dendritic cells in the induction of anti-tumor immune responses, the number of dendritic cells that can be isolated from experimental animals is limiting and often precludes in-depth analyses of these cells. To overcome this limitation, dendritic cell lines have been established and have facilitated the experimental study of dendritic cell biology. In this study we compare the dendritic cell lines DC2.4 and JAWSII as in vitro model systems for studying the influence of melanoma-derived factors on dendritic cell maturation and activation. Using flow cytometry and ELISA analyses, we evaluate the expression of costimulatory/MHC class II molecules and proinflammatory cytokines/chemokines by these dendritic cell lines in their resting state and following LPS stimulation in the presence or absence of B16-F1 melanoma-derived factors. Results: We demonstrate that soluble B16-F1-derived factors suppress the LPSinduced upregulation of CD40, CD80, CD86 and MHC class II on both the DC2.4 and JAWSII dendritic cell lines. Interestingly, LPS-induced secretion by DC2.4 cells of the proinflammatory cytokines/chemokines TNF- $\alpha$, IP-10, MIP-1 $\alpha$, MIP-1 $\beta$ and MCP-1 is also altered by B16-F1-derived factors, whereas JAWSII cell cytokine/chemokine production is affected to a lesser extent by such factors, with only IL-1 $\beta$ and IP-10 production being suppressed. Conclusions/Recommendations: We conclude that melanoma-derived factors can suppress dendritic cell maturation/activation and that the DC2.4 and JAWSII dendritic cell lines are effective in vitro models for future studies that aim to (1) identify factors that influence both the susceptibility and the resistance of dendritic cells to tumor-mediated immunosuppression and (2) investigate the influence of tumor-altered dendritic cells on the quality of anti-tumor $\mathrm{T}$ cell responses.
\end{abstract}

Keywords: Melanoma, Tumor Immunity, Dendritic Cell

\section{INTRODUCTION}

Dendritic Cells (DC) are a population of lymphoidand peripheral tissue-resident innate immune cells derived from lymphoid and myeloid bone marrow progenitors. DC possess a number of immunoregulatory functions and are capable of inducing either tolerance to or activation against the antigens they encounter. Importantly, the immune response elicited by DC to such antigens is dictated by the context in which these antigens are acquired and the maturation and activation status of the dendritic cell (Matzinger, 1994; Banchereau and Steinman, 1998; Janeway and Medzhitov, 2002).
Immature DC induce either immunologic ignorance or tolerance to self antigens and antigens derived from sources that do not represent "danger" to the host. On the other hand, acquisition of antigens in the presence of "danger signals" that stimulate DC maturation and activation induces DC to express costimulatory molecules and proinflammatory cytokines and chemokines that are necessary to activate and recruit $\mathrm{T}$ cells and other immune effectors to the source of antigen within the body for elimination from the host (Albert et al., 2001; Filatenkov et al., 2006; Sousa, 2006; Joffre et al., 2009). Because DC maturation and activation are so critical for the induction of immunity, 
it is important to gain a better understanding of the factors that regulate these processes and the DC functions associated with them.

Immunity to cancer has long been appreciated (Broek et al., 1996; Shankaran et al., 2001) and since the emergence of the field of tumor immunology, several studies have subsequently described mechanisms of tumor immune escape to explain the outgrowth of tumors in immunocompetent hosts (Rabinovich et al., 2007). The majority of these studies report mechanisms of escape from tumor antigenspecific $\mathrm{T}$ cells, either by evasion of activated, effector $\mathrm{T}$ cells (through dowregulation of tumor antigen/MHC molecule expression (Restifo et al., 1993; Garrido et al., 1995) or induction of T cell death (Hahne et al., 1996; Dong et al., 2002) or by suppression of effector $\mathrm{T}$ cell function (Radoja et al., 2001; Blohm et al., 2002; Mortarini et al., 2003; Anichini et al., 2003; Whiteside et al., 2004; Koneru et al., 2005; Hargadon et al., 2006). Because of the importance of DC in regulating immune responses, it is appealing to speculate that tumors might also interfere with DC function. However, while several studies have identified an immunosuppressive role for DC-like precursors known as tumor-associated immature myeloid cells (Almand et al., 2001; Terabe et al., 2003; Kusmartsev et al., 2004; Huang et al., 2006; Ostrand-Rosenberg and Sinha, 2009), little is currently known about the influence of tumors on the function of fully differentiated DC. This lack of information is due in large part to the limiting numbers of DC in situ and the associated difficulties inherent in isolating these cells from tumor-bearing patients and animals for experimental analyses. Importantly, though, DC lines that can be maintained in vitro have recently been established to overcome these limitations and facilitate the study of DC immunobiology (Shen et al., 1997; MacKay and Moore, 1997; Helden et al., 2008). Using the C57B1/6-derived DC2.4 cell line, we have previously reported that B16-F1 melanoma-derived factors are capable of suppressing the LPS-induced expression of costimulatory molecules and proinflammatory cytokines/chemokines by these cells and that the extent of this suppression correlates with the tumorigenicity of the melanoma variant under study (Hargadon et al., 2012). In this report, we compare the melanoma-induced suppression of the DC2.4 cell line with that of another commonly used C57Bl/6-derived DC line, JAWSII, in order to establish the efficacy of these lines as in vitro model systems for studying melanoma-associated suppression DC maturation and activation and to determine whether the quality of this immunosuppression is dependent on the DC line under study. The significance of our results and the utility of these DC lines as models for studying melanoma-associated suppression of DC function are discussed herein.

\section{MATERIALS AND METHODS}

\subsection{Cell Lines}

DC2.4 cells are an adherent C57B1/6 bone marrowderived DC line and were kindly provided by Dr. Kenneth Rock (Dana Farber Cancer Institute, Boston, MA). The JAWSII cell line was purchased from American Type Culture Collection (Manassas, VA) and is a GM-CSFdependent, $\mathrm{C} 57 \mathrm{~B} 1 / 6$ bone marrow-derived $\mathrm{DC}$ line that grows as a mixture of adherent and suspension cells. B16F1 murine melanoma cells were a generous gift of Dr. Victor Engelhard (University of Virginia). The DC2.4 and B16-F1 cell lines were grown in RPMI-1640 medium (Thermo Scientific, Hudson, NH) supplemented with 10\% fetal bovine serum (Premium Select, Atlanta Biologicals, Norcross, GA), $2 \mathrm{mM}$ L-glutamine, $2 \mathrm{~g} \mathrm{~L}^{-1}$ glucose, $2 \mathrm{~g} \mathrm{~L}^{-1}$ sodium bicarbonate and a mixture of $100 \mathrm{U} / \mathrm{mL}$ penicillin and $100 \mu \mathrm{g} / \mathrm{mL}$ streptomycin (ATCC). JAWSII cells were grown in HyClone Iscove's Modified Dulbecco's Medium (Thermo Scientific) supplemented with 10\% fetal bovine serum (Premium Select, Atlanta Biologicals), $4 \mathrm{mM} \mathrm{L-}$ glutamine, HEPES, $0.1 \mathrm{mM} \beta$-mercaptoethanol (Sigma, St. Louis, MO), $1 \mathrm{mM}$ sodium pyruvate (Sigma), a mixture of $100 \mathrm{U} / \mathrm{mL}$ penicillin and $100 \mu \mathrm{g} \mathrm{mL}^{-1}$ streptomycin (ATCC) and $5 \mathrm{ng} / \mathrm{mL}$ recombinant murine GM-CSF (Thermo Scientific). Cells were passaged at $80-90 \%$ confluence.

\subsection{Antibodies and Reagents}

The following monoclonal antibodies were purchased from eBioscience (San Diego, CA) and used in the analysis of DC2.4 and JAWSII maturation: anti-CD40 PE (1C10), anti-CD80 PE (16-10A1), anti-CD86 PE (GL1) and antiMHC class II (I-A/I-E) PE (M5/114.15.2). LPS was purchased from Invivogen (San Diego, CA).

\subsection{Generation of Tumor-Conditioned Media (TCM)}

B16-F1 tumor cells $\left(1 \times 10^{6}\right.$ cells/well $)$ were plated in 6well tissue culture plates. Twenty four hours later, culture supernatants were collected and centrifuged at $1,000 \mathrm{~g}$ for 10 min to remove any cellular debris. The resulting supernatant was used as TCM for experiments as indicated.

\subsection{Maturation/Activation of DC Lines}

DC2.4 or JAWSII cells growing in culture were harvested, resuspended in appropriate growth media \pm 1 
$\mu \mathrm{g} \mathrm{mL}^{-1}$ LPS and plated at $1 \times 10^{6}$ cells $/ \mathrm{mL}$ in 6-well tissue culture plates. In some experiments, DC were resuspended in $\mathrm{TCM} \pm 1 \mu \mathrm{g} \mathrm{mL}^{-1}$ LPS. After $24 \mathrm{~h}$, cells were collected by cell scraping and fixed for flow cytometry staining. At this time, cell culture supernatants were also collected by centrifugation at $1,000 \mathrm{~g}$ to remove any cellular debris and frozen at $-20^{\circ} \mathrm{C}$ for subsequent ELISA analysis of cytokine and chemokine production.

\subsection{Flow Cytometry Analysis}

Cells were collected from in vitro cultures and fixed in $2 \%$ paraformaldehyde for $10 \mathrm{~min}$ at room temperature. Cells were then resuspended in FACS Buffer (PBS supplemented with 0.5\% BSA and 0.02\% $\mathrm{NaN}_{3}$ ) and $\mathrm{Fc}$ blocked for $5 \mathrm{~min}$ at $4^{\circ} \mathrm{C}$, followed by surface staining with the indicated monoclonal antibodies for $30 \mathrm{~min}$ at $4{ }^{\circ} \mathrm{C}$ in the dark. Labeled cells were detected by flow cytometry using a FACS Canto (BD, Mountain View, CA) and were analyzed using Flowjo software (Tree Star).

\subsection{ELISA}

ELISA analysis for the indicated cytokines and chemokines was performed using Ready-Set-Go! ELISA kits from eBioscience (San Diego, CA) and SABiosciences ELISArray kits from Qiagen (Frederick, $\mathrm{MD)}$ according to the manufacturers' protocols.

\subsection{Statistical Analyses}

Values are expressed as the mean \pm SD and differences among indicated groups were analyzed using unpaired $t$ tests. A value of $p \leq 0.05$ was considered significant and is represented in graphs by $*$. ** = $\mathrm{p} \leq 0.01, * * *=\mathrm{p} \leq 0.001, * * * *=\mathrm{p} \leq 0.0001$.

\section{RESULTS}

\subsection{The Influence of B16-F1 Melanoma-Derived Factors on LPS-Induced Maturation of DC2.4 and JAWSII DC Lines}

B16-F1 is a highly aggressive murine melanoma that grows progressively and metastasizes upon tumor challenge of mice (Fidler, 1975). This tumor has also been shown to induce dysfunctional anti-tumor $\mathrm{CD}^{+} \mathrm{T}$ cell responses (Hargadon et al., 2006) that are similar to those often observed in melanoma patients (Mortarini et al., 2003; Anichini et al., 2003; Zippelius et al., 2004) and it has been suggested that this dysfunction may arise from an influence of the tumor on the functional quality of
DC. Because of the inherent difficulties in isolating tumor-associated DC from B16-F1 tumor-bearing animals, though, it has thus far not been possible to thoroughly examine the nature of these cells. Therefore, we wished to employ an in vitro model system to directly study the effects of B16-F1-derived factors on DC maturation and activation. To this end, we stimulated DC2.4 and JAWSII DC lines with LPS in either normal growth media or B16-F1 tumor-conditioned media (TCM) for $24 \mathrm{~h}$. In the absence of B16-F1-derived factors, LPS induced both DC lines to upregulate their expression of MHC class II and the costimulatory molecules CD40, CD80 and CD86 (Fig. 1).

Interestingly, when both the DC2.4 and JAWSII DC lines were stimulated with LPS in the presence of B16F1 TCM, the upregulation of MHC class II, CD40, CD80 and CD86 was suppressed (Fig. 1). These data demonstrate that soluble factors derived from B16-F1 melanoma suppress the maturation of $\mathrm{DC} 2.4$ and JAWSII DC.

\subsection{The Influence of B16-F1 Melanoma-Derived Factors on LPS-Induced Proinflammatory Cytokine/Chemokine Expression by DC2.4 and JAWSII DC Lines}

Our previous data regarding LPS-induced maturation of the DC2.4 and JAWSII cell lines confirm findings from other studies (He et al., 2007; Rhule et al., 2008; Jiang et al., 2008) demonstrating that these cells are relatively immature in their resting state and can be induced to upregulate expression of costimulatory and MHC class II molecules. However, an in-depth characterization of the cytokines and chemokines produced by resting and LPS-stimulated DC2.4 and JAWSII cells has yet to be reported. Therefore, in order to assess the cytokine and chemokine profiles of these DC lines, cells were left untreated or were exposed to LPS for $24 \mathrm{~h}$ and cell culture supernatants were then collected for ELISArray analysis of a panel of cytokines (Fig. 2A) and chemokines (Fig. 2B). Following LPS stimulation, both DC2.4 and JAWSII DC upregulated their expression of the proinflammatory cytokines IL$1 \alpha$, IL- 6 and TNF- $\alpha$. Additionally, the JAWSII cell line also upregulated expression of IL-1 $\beta$ after stimulation with LPS. Likewise, LPS stimulation induced both cell lines to increase their expression of a number of chemokines, including RANTES, MCP-1, MIP-1 $\alpha$, MIP-1 $\beta$ and IP-10. 


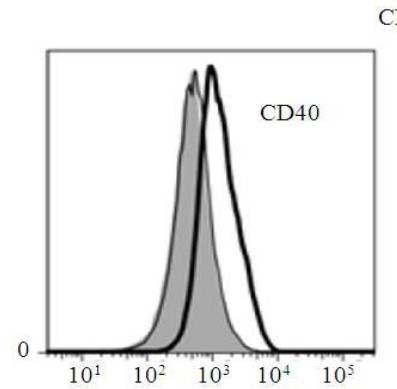

CD2.4
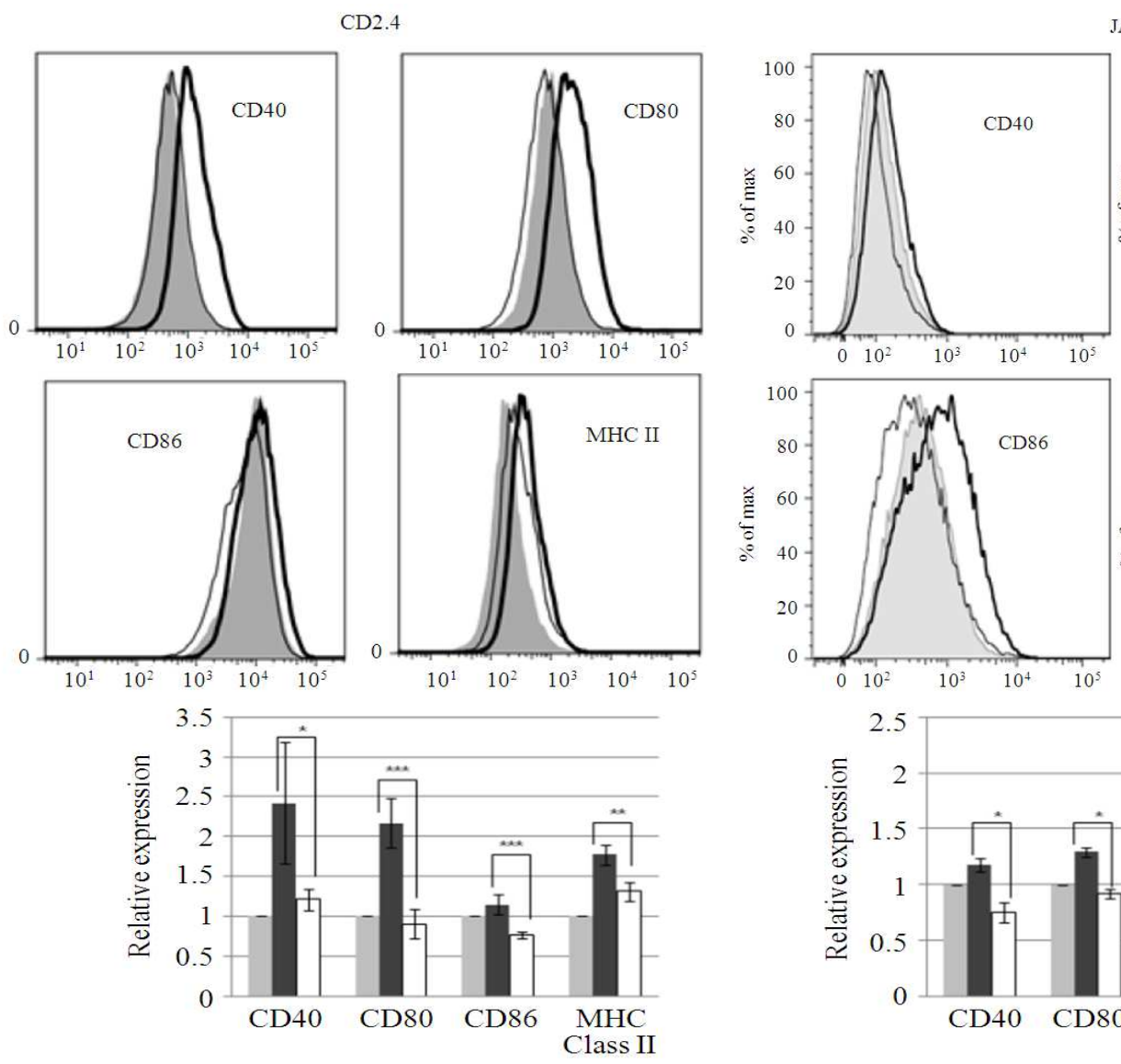

JAWSII
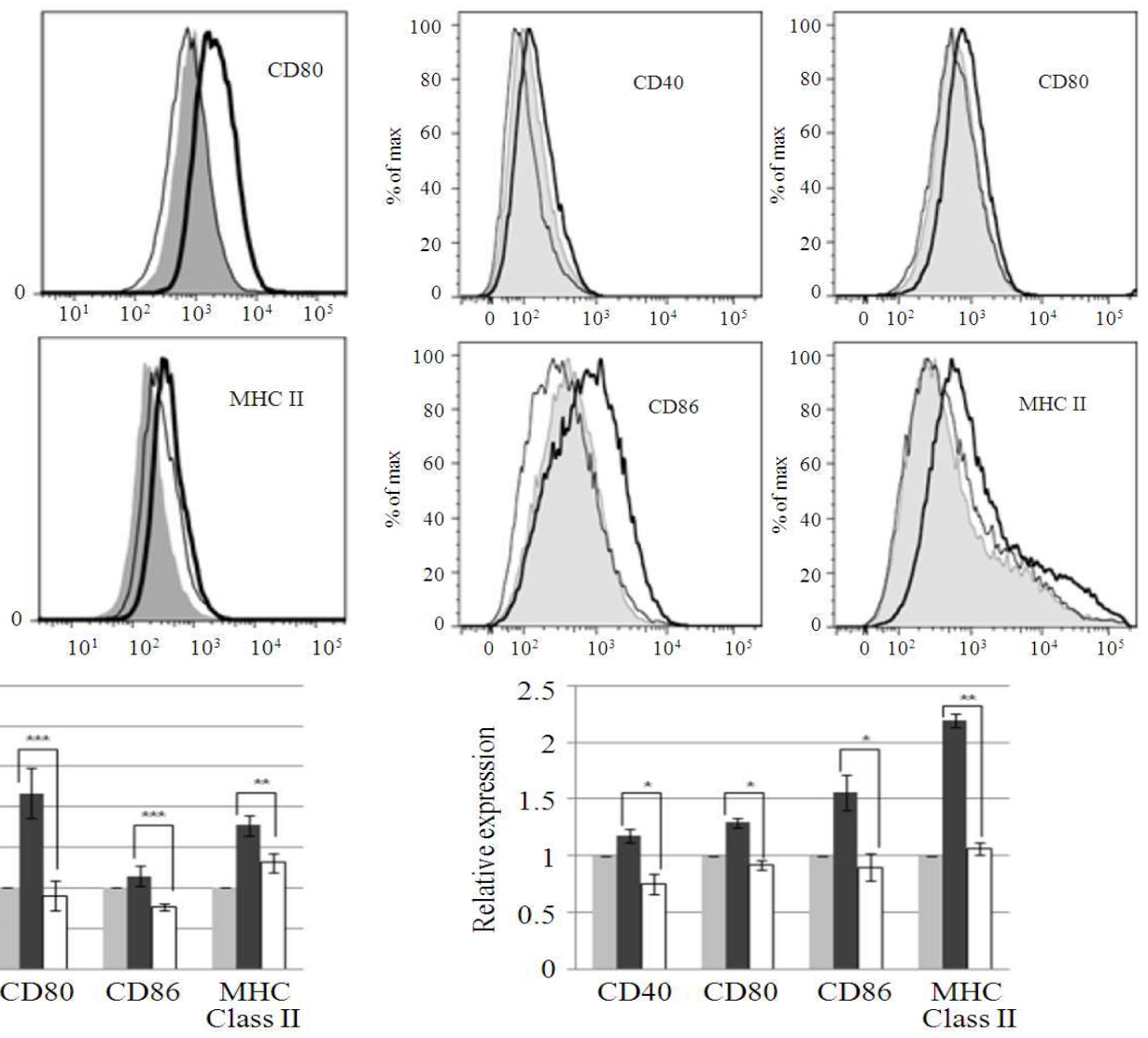

Untreated

LPS

LPS + B 16-F 1

Fig. 1. B16-F1 tumor-altered maturation of DC2.4 and JAWSII DC. DC2.4 (A) and JAWSII (B) cells were cultured for $24 \mathrm{~h}$ in normal growth media (shaded histogram), growth media containing $1 \mu \mathrm{g} \mathrm{mL}{ }^{-1}$ LPS (bold-lined histogram), or TCM derived from B16-F1 supplemented with $1 \mu \mathrm{g} \mathrm{mL}{ }^{-}$LPS (thin-lined histogram). Following culture, cells were harvested and maturation status was assessed by staining with the indicated antibodies for flow cytometric analysis. Relative expression was calculated as the fold change in the geometric mean of fluorescence as compared to untreated cells. Histogram data are representative of 4 independent experiments and the bar graph depicts the mean \pm SD of these 4 independent experiments

Because B16-F1-derived factors suppressed LPSinduced costimulatory/MHC class II molecule expression by DC2.4 and JAWSII DC, we wished to determine whether tumor-derived factors might also alter cytokine and chemokine production by these cells. Therefore, we stimulated DC2.4 and JAWSII DC lines with LPS in either normal growth media or B16-F1 TCM for $24 \mathrm{~h}$ and performed quantitative ELISA analysis on cell culture supernatants to assess production of the cytokines/chemokines identified in the ELISArray reported in Fig. 2. As shown in Fig. $3 \mathbf{A}, \quad$ DC2.4 production of several cytokines/chemokines was altered when these cells were stimulated with LPS in the presence of B16-F1 TCM. TNF- $\alpha$ production was augmented when these cells were exposed to soluble factors derived from the B16-F1 melanoma. These tumor-derived factors also suppressed the LPS-induced secretion of the chemokines IP-10, MCP-1, MIP-1 $\alpha$ and MIP-1 $\beta$ by DC2.4 cells. Interestingly, LPS-induced cytokine/chemokine secretion by JAWSII DC was affected to a lesser extent by B16-F1 tumor-derived factors (Fig. 3B). With the exception of suppressed IL-1 $\beta$ and IP-10 production, JAWSII cytokine and chemokine secretion patterns were not altered when these cells were stimulated with LPS in the presence of B16-F1 TCM. 
Kristian Michael Hargadon et al. / American Journal of Immunology, 8 (4) (2012) 179-190

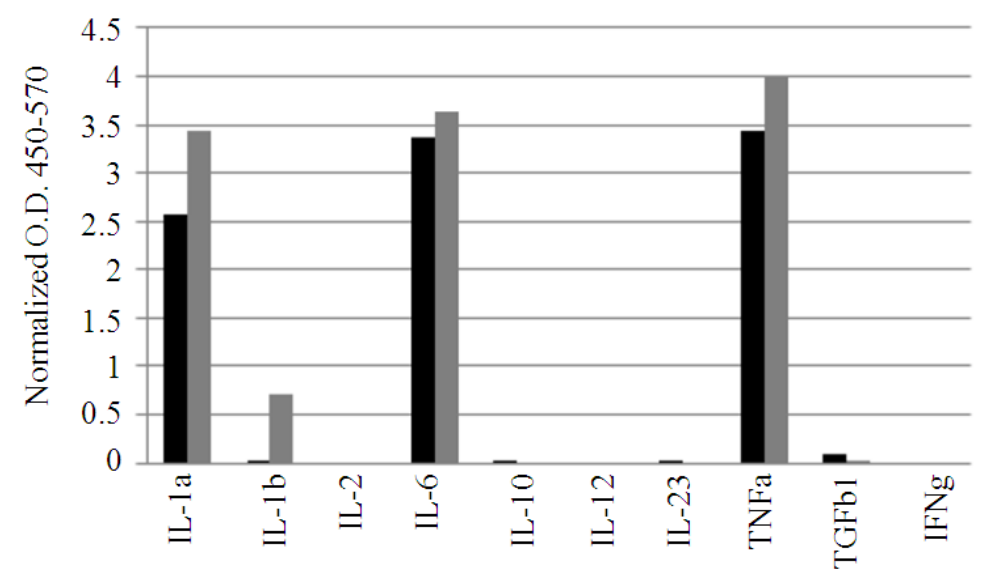

(A)

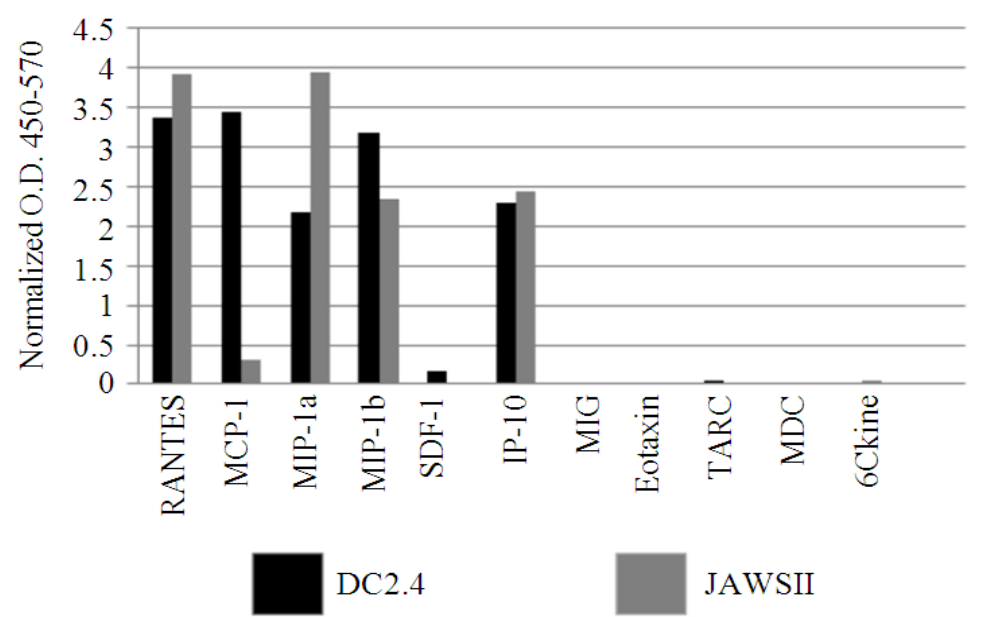

(B)

Fig. 2. LPS-induced cytokine/chemokine secretion by DC 2.4 and JAWSII DC. DC 2.4 and JAWSII cells were cultured for $24 \mathrm{~h}$ in normal growth media in the presence/absence of $1 \mu \mathrm{g} \mathrm{mL} \mathrm{m}^{-1}$ LPS. Following culture, supernatants were collected for analysis by ELISArray to detect expression of a panel of cytokines (A) and chemokines (B). Normalized data represent the O.D. 450570 values of LPS-stimulated cells with the O.D. 450-570 values of untreated cells subtracted out.
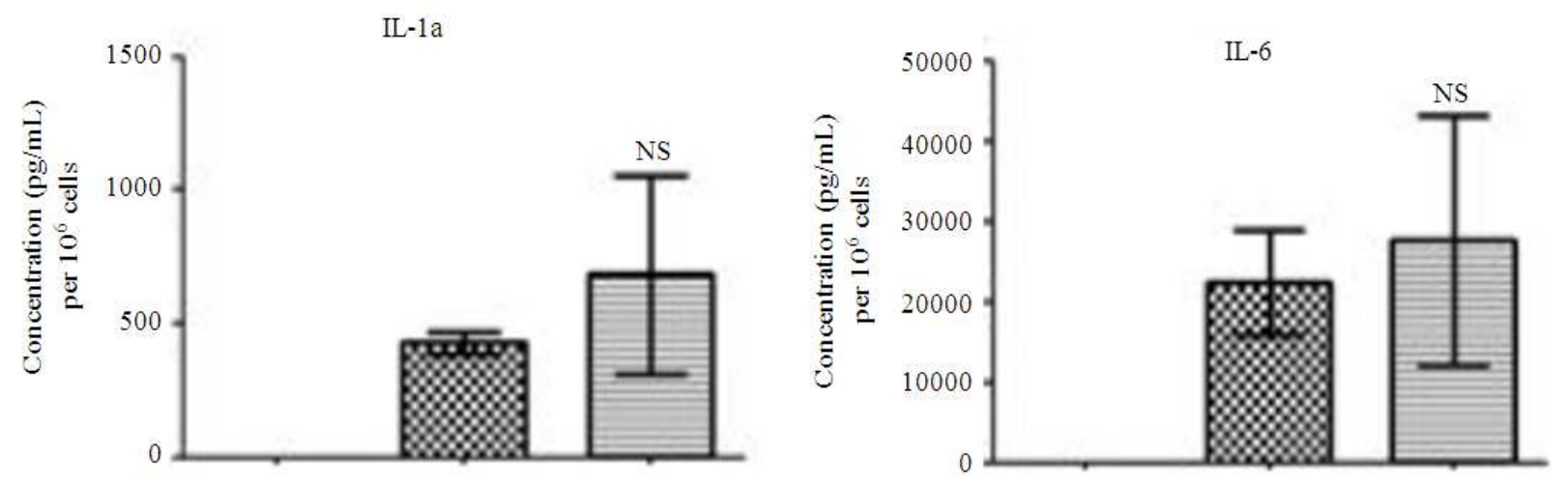
Kristian Michael Hargadon et al. / American Journal of Immunology, 8 (4) (2012) 179-190
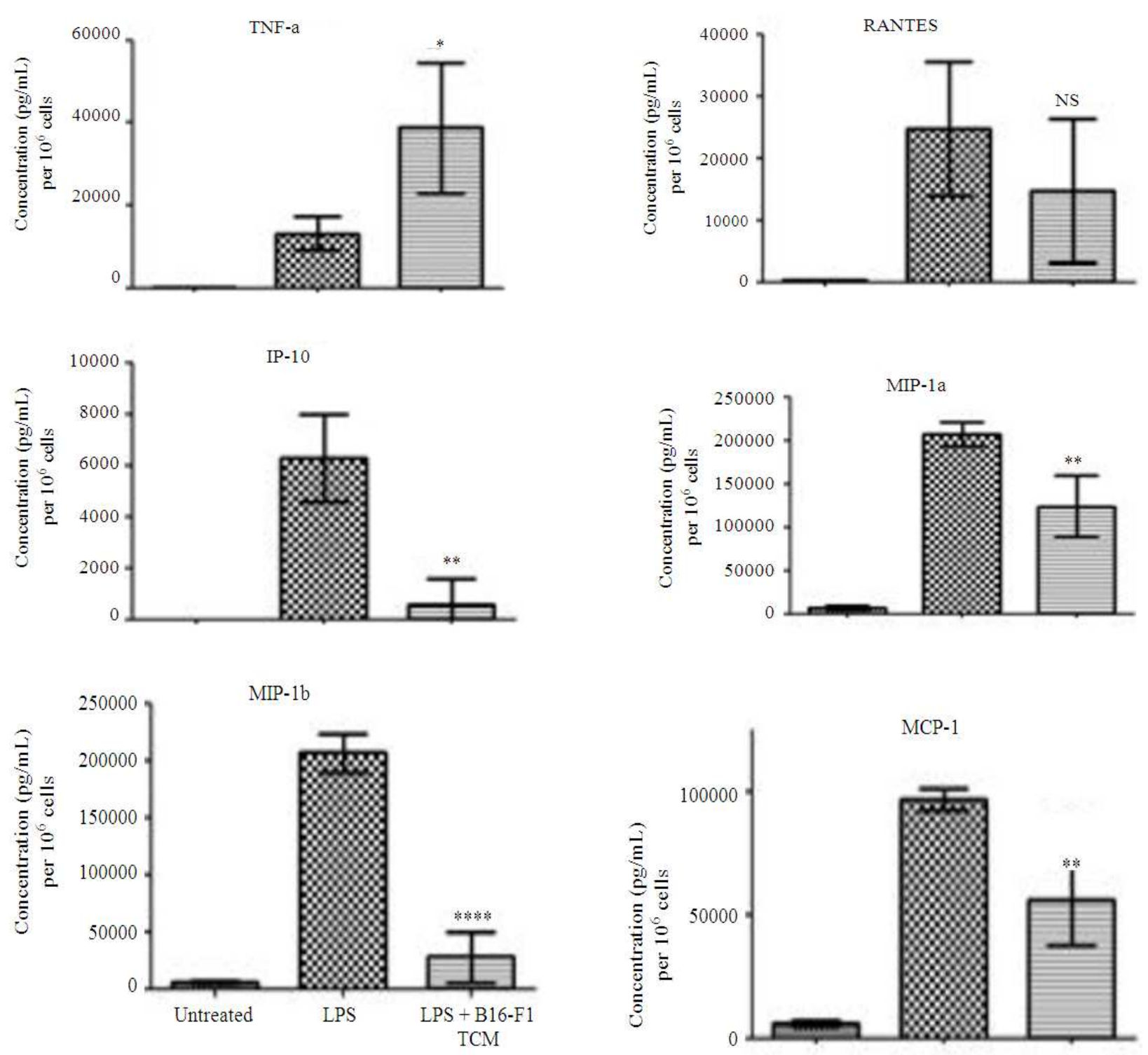

(A)
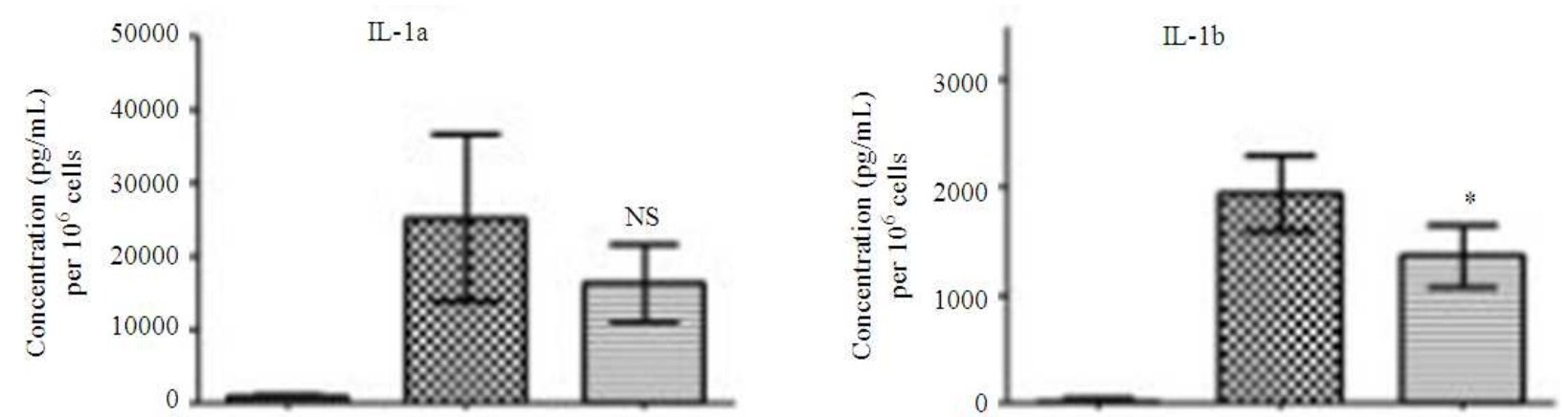
Kristian Michael Hargadon et al. / American Journal of Immunology, 8 (4) (2012) 179-190
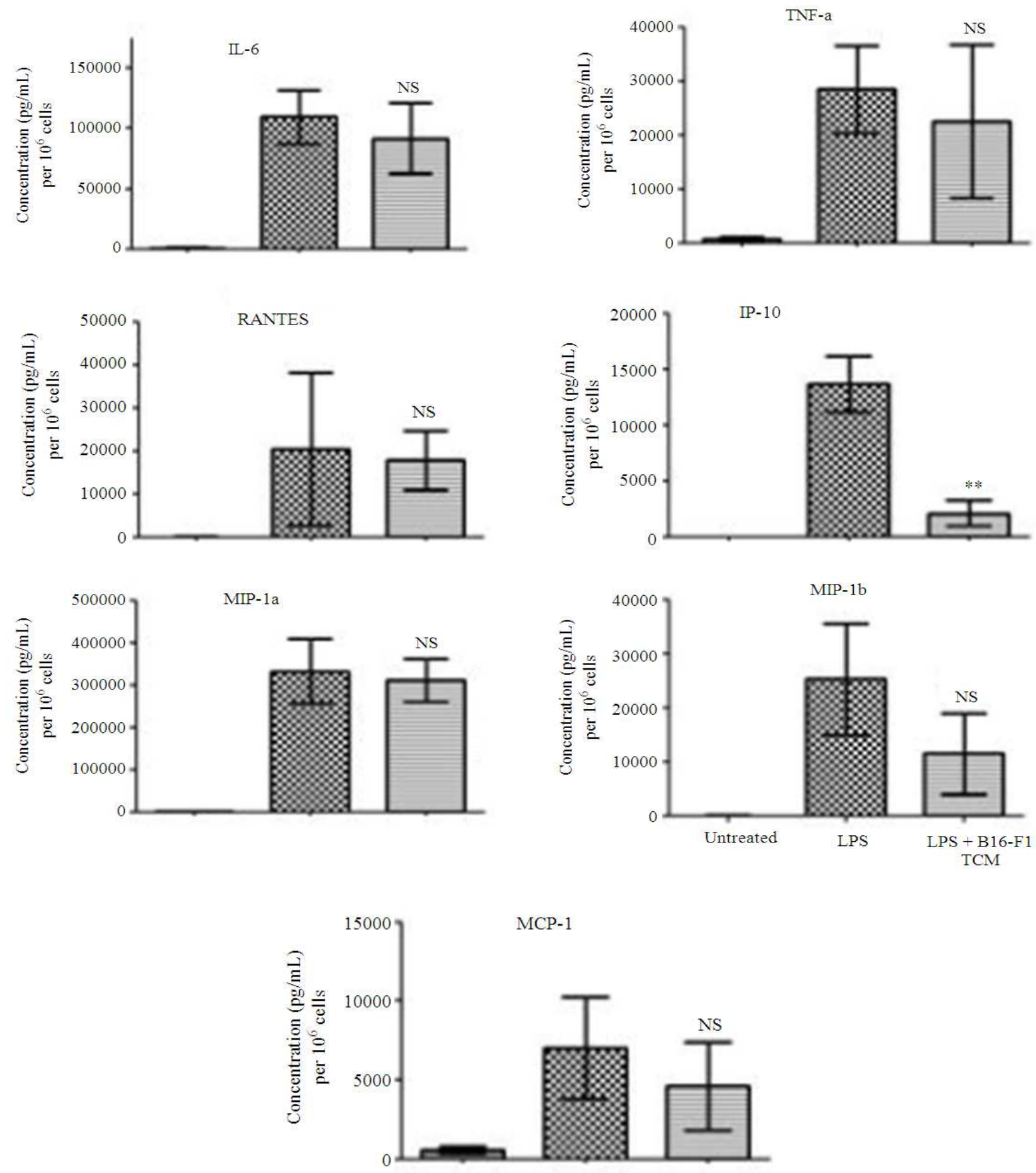

(B)

Fig. 3. B16-F1 tumor-altered cytokine/chemokine secretion by LPS-stimulated DC2.4 and JAWSII DC. DC2.4 (A) and JAWSII (B) cells were cultured for $24 \mathrm{~h}$ in normal growth media or in TCM derived from B16-F1 cultures in the presence/absence of 1 $\mu \mathrm{g} \mathrm{mL} \mathrm{m}^{-1}$ LPS. Following culture, supernatants were collected for quantitative ELISA analysis of cytokines/chemokines identified previously by ELISArray. No cytokines/chemokines presented were detected in B16-F1 TCM alone. Data are expressed as the mean \pm SD of 4 independent experiments 
Importantly, all of the cytokines and chemokines detected in these experiments were produced by the DC lines; these molecules were not produced by B16-F1 melanoma cells as determined by ELISA analysis of TCM alone (data not shown). Collectively, these data demonstrate that B16-F1 melanoma-derived factors are capable of suppressing DC maturation and activation and that the DC2.4 and JAWSII dendritic cell lines are each effective in vitro models for studying melanomaassociated suppression of different cytokine/chemokine signaling pathways.

\section{DISCUSSION}

DC maturation and activation are critical processes that determine DC function and shape overall innate and adaptive immune responses within a host. Because of the central role played by DC in dictating the outcome of host immunity to a particular source of antigen, it is important to understand the factors that regulate their maturation and activation. In addition, although several mechanisms of tumor immune evasion have been described (Rabinovich et al., 2007), the influence of tumors on DC maturation and activation and the role of DC in determining successful anti-tumor immunity versus tumor immune escape have yet to be extensively studied. In this report, we utilize an in vitro model system to evaluate the influence of B16-F1 melanomaderived factors on the LPS-induced maturation and activation of two DC lines, DC2.4 and JAWSII. Both of these cells lines are relatively immature in their resting state, expressing low levels of MHC class II and costimulatory molecules and producing little, if any, of a number of cytokines and chemokines. Both cell lines are matured and activated by LPS stimulation, which upregulates expression of MHC class II, costimulatory molecules and proinflammatory cytokines and chemokines. We demonstrate that soluble tumor-derived factors from B16-F1 melanoma suppress the LPSinduced upregulation of costimulatory and MHC class II molecules on both the DC2.4 and JAWSII DC lines. Additionally, these tumor-derived factors alter LPSinduced secretion of several proinflammatory cytokines and chemokines by DC2.4 cells, while JAWSII cytokine/chemokine production, with the exception of IL-1 $\beta$ and IP-10, is unaffected by B16-F1-derived factors. Collectively, these results indicate that melanoma-derived factors can suppress dendritic cell maturation/activation and that the DC2.4 and JAWSII dendritic cell lines are each effective in vitro models for studying different aspects of this phenomenon. Furthermore, because of the role of DC in the induction of both innate and adaptive immunity, our data also suggest that the interaction between DC and tumors is likely a critical factor in determining the overall quality of an anti-tumor immune response.

Anti-tumor immune responses are frequently detected in melanoma patients. In some cases, functional $\mathrm{CD} 8^{+} \mathrm{T}$ cell responses directed against melanocyte differentiation antigens correlate with tumor regression and enhanced survival (Rosenberg and White, 1996; Yamshchikov et al., 2001). However, in many cases dysfunctional $\mathrm{CD}^{+} \mathrm{T}$ cells are recovered from tumors and tumor-draining lymph nodes of melanoma patients (Mortarini et al., 2003; Anichini et al., 2003; Zippelius et al., 2004). It has recently been reported in a preclinical B16-F1 murine melanoma model that $\mathrm{CD}^{+} \mathrm{T}$ cell responses induced against the highly conserved tyrosinase-derived $\mathrm{Tyr}_{369}$ antigen (a well-characterized human tumor antigen) are also dysfunctional, exhibiting robust proliferation but minimal effector function (Hargadon et al., 2006). The basis for this melanoma antigen-specific $\mathrm{CD} 8^{+} \mathrm{T}$ cell dysfunction in both patients and animals has remained unclear. While it is possible that tumors or tumor-derived factors directly suppress effector $\mathrm{CD}^{+} \mathrm{T}$ cell differentiation, it has been suggested that this $\mathrm{T}$ cell dysfunction might also arise from an influence of the tumor on cross-presenting DC (Hargadon et al., 2006; Ferguson et al., 2008). This latter possibility has been difficult to address by either in vivo or ex vivo analyses due to the limiting number of DC that can be isolated from melanoma patients or tumor-bearing animals. Surprisingly, despite the utility of DC lines as in vitro models for studying DC function, only one other group has examined the influence of a tumor on the maturation and activation of a DC line (Alshamsan et al., 2010). In their study, melanoma-derived factors actually enhanced LPS-induced expression of select costimulatory molecules on DC2.4 cells, although cytokine and chemokine profiles were not assessed. Importantly, that previous study evaluated the effects of TCM derived from B16-F10, a different variant of B16 melanoma than the $\mathrm{B} 16-\mathrm{F} 1$ line that is associated with dysfunctional $\mathrm{CD}^{+} \mathrm{T}$ cell responses in vivo (Hargadon et al., 2006) and that suppresses DC maturation and activation in our current study. Additionally, we have recently demonstrated that a chemically mutated variant of B16, the poorly tumorigenic D5.1G4 melanoma, is significantly less immunosuppressive than B16-F1 with respect to the influence of tumor-derived factors on DC2.4 maturation and activation (Hargadon et al., 2012). We have also observed that B16-F1 secretes significantly greater amounts of the immunosuppressive cytokines TGF $\beta 1$ and VEGF-A than does D5.1G4 (unpublished data) and we are currently exploring the roles of these tumor-derived factors in the suppression of DC2.4 
maturation and activation. It is therefore very likely that differences in these or other soluble factors derived from B16 melanoma variants account for the different effects of melanoma-derived factors on DC maturation and activation observed in our current and previous studies and the study by Alshamsan et al. (2010). Based on earlier studies that document the B16-F1-associated dysfunction of tumor Ag-specific $\mathrm{CD}^{+} \mathrm{T}$ cells (Hargadon et al., 2006; Ferguson et al., 2008) and our current observations that B16-F1-derived factors suppress the maturation and activation of DC2.4 and JAWSII cells, it will be interesting to determine whether these B16-F1-altered DC lines induce the type of incomplete $\mathrm{CD}^{+} \mathrm{T}$ cell differentiation associated with this tumor in vivo. We believe that this in vitro model offers a nice system for investigating the impact of tumor-altered DC on the quality of anti-tumor immune responses and we are currently exploring how these tumor-altered DC influence both $\mathrm{CD} 8^{+}$cytotoxic and $\mathrm{CD} 4^{+}$helper $\mathrm{T}$ cell activation and differentiation.

The LPS-induced secretion of a number of chemokines (RANTES, MCP-1, MIP- $1 \alpha$, MIP-1 $\beta$ and IP$10)$ by the DC 2.4 and JAWSII DC lines is an intriguing finding. Interestingly, it has been shown that human monocyte-derived DC also produce IP-10 following LPS stimulation (Re and Strominger, 2001). IP-10 plays an important role in the chemoattraction of Th1 helper $\mathrm{T}$ cells, which express high levels of the CXCR3 receptor (Sallusto et al., 1998). It is interesting to speculate that recruitment of such Th1 cells into a tumor is likely to improve the activation of tumor-infiltrating $\mathrm{CD}^{+} \mathrm{T}$ cells and that suppression of IP-10 production by tumorassociated DC might lead to a diminished anti-tumor $\mathrm{T}$ cell response. The suppressive activity of B16-F1-derived factors on LPS-induced IP-10 production by DC2.4 and JAWSII cells therefore has potentially significant implications for the quality of anti-tumor $\mathrm{T}$ cell responses. Additionally, we also observed significant suppression of MCP-1, MIP-1 $\alpha$ and MIP-1 $\beta$ production by B16-F1influenced DC2.4 cells and these chemokines regulate the migration of a number of immune cells that include monocytes, macrophages, NK cells and T cells (Olson and Ley, 2002). Melanoma-associated interference with the production of these chemokines by DC may therefore impact the recruitment of many immune effectors to the tumor and it will be interesting to compare in future studies the immune infiltration of B16-F1 melanoma with that of melanoma variants that do not suppress DC chemokine production as efficiently. Such a comparison will allow for the correlation of immune cell infiltration of a tumor with the efficacy of the anti-tumor immune response and may suggest strategies for targeting the particular immune effector types associated with greater tumor control to the tumor.
A variety of factors that are secreted by many tumors have been shown to interfere with DC function (Yang and Carbone, 2004; Fricke and Gabrilovich, 2006; Lin et al., 2010) and melanomas in particular secrete a number of soluble factors that modulate immune responses (Ilkovitch and Lopez, 2008). B16-F1 melanoma secretes bioactive TGF- $\beta 1$ (Peter et al., 2001; our data not shown] and this immunosuppressive cytokine can inhibit costimulatory molecule expression as well as cytokine production by DC (Yamaguchi et al., 1997; Geissmann et al., 1999). B16-F1 also secretes VEGF-A but does not produce IL-10 (unpublished data) and it remains unknown whether this melanoma secretes other immunoregulatory factors. We are currently investigating the profile of immunosuppressive proteins secreted by B16-F1 so that we may test these molecules both individually and collectively for their role in the B16-F1-associated suppression of DC maturation and activation. Conversely, we also hope to gain mechanistic insights into B16-F1-induced DC immunosuppression by comparing the cell surface receptor expression of DC2.4 and JAWSII DC. Because JAWSII DC are less susceptible than DC2.4 cells to B16-F1-mediated suppression of chemokine production, it will be interesting to determine whether there are immunoregulatory receptors uniquely expressed by DC2.4 cells that increase their susceptibility to immunosuppressive factors. This information may shed light on the cellular targets of B16-F1-derived factors and ultimately point to the mechanism(s) by which this tumor suppresses DC function. Identification of the tumor-derived factors responsible for DC immunosuppression and their targets will suggest strategies for interfering with tumor-associated DC dysfunction that may improve the functional quality of endogenous DC in cancer patients as well as the efficacy of exogenous DC-based anti-tumor vaccines.

\section{CONCLUSION}

The DC lines DC2.4 and JAWSII are useful models for the study of factors that regulate DC maturation and activation. Tumor-derived factors from B16-F1 melanoma suppress the LPS-induced upregulation of costimulatory and MHC class II molecules on both of these DC lines. Conversely, LPS-induced proinflammatory cytokine and chemokine production by these two DC lines is differentially regulated by B16-F1derived factors. Therefore, both the DC2.4 and JAWSII DC lines will serve as useful tools for future investigations of the mechanism of melanoma-associated suppression of DC maturation and activation as well as the basis for resistance versus susceptibility of DC to such immunosuppression. 


\section{ACKNOWLEDGEMENT}

This study was supported by a Virginia Academy of Science Jeffress Research Grant from the Thomas F. Jeffress and Kate Miller Jeffress Memorial Trust and a Virginia Foundation for Independent Colleges Mednick Memorial Fellowship to K. Hargadon, a Virginia Foundation for Independent Colleges Undergraduate Science Research Fellowship to Y. Ararso and O. Forrest and a Hampden-Sydney College Research Grant from the Arthur Vining Davis endowment. We would like to thank the University of Virginia Beirne B. Carter Center for Immunology Research for access to their flow cytometer.

The authors declare that the research was conducted in the absence of any commercial or financial relationships that could be construed as a potential conflict of interest.

\section{REFERENCES}

Albert, M.L., M. Jegathesan and R.B. Darnell, 2001. Dendritic cell maturation is required for the crosstolerization of $\mathrm{CD}^{+} \mathrm{T}$ cells. Nat. Immunol., 2: 1010-1017. PMID: 11590405

Almand, B., J.I. Clark, E. Nikitina, B.J. Van and N.R. English et al., 2001. Increased production of immature myeloid cells in cancer patients: A mechanism of immunosuppression in cancer. J. Immunol., 166: 78-689. PMID: 11123353

Alshamsan, A., S. Hamdy, S. Das, A. Lavasanifar and J. Samuel et al., 2010. Validation of bone marrow derived dendritic cells as an appropriate model to study tumor-mediated suppression of DC maturation through STAT3 hyperactivation. J. Pharm. Pharmaceut. Sci., 13: 21-26. PMID: 20456827

Anichini, A., A. Scarito, A. Molla, G. Parmiani and R. Mortarini, 2003. Differentiation of CD8+ T cells from tumor invaded and tumor-free lymph nodes of melanoma patients: Role of common gamma-chain cytokines. J. Immunol., 171: 2134-2141. PMID: 12902520

Banchereau, J. and R.M. Steinman, 1998. Dendritic cells and the control of immunity. Nature, 392: 245-252. PMID: 9521319

Blohm, U., E. Roth, K. Brommer, T. Dumrese and F.M. Rosenthal et al., 2002. Lack of effector cell function and altered tetramer binding of tumorinfiltrating lymphocytes. J. Immunol., 169: 55225530. PMID: 12421928
Broek, L.V.D., D. Kagi, F. Ossendorp, R. Toes and S. Vamvakas et al., 1996. Decreased tumor surveillance in perforin-deficient mice. J. Exp. Med., 184: 1781-1790. PMID: 8920866

Dong, H., S.E. Strome, D.R. Salomao, H. Tamura and F. Hirano et al., 2002. Tumor-associated B7-H1 promotes T-cell apoptosis: A potential mechanism of immune evasion. Nat. Med., 8: 793-800. PMID: 12091876

Ferguson, A.R., L.A. Nichols, A.L. Zarling, E.D. Thompson and C.C. Brinkman et al., 2008. Strategies and challenges in eliciting immunity to melanoma. Immunol. Rev., 222: 28-42. PMID: 18363993

Fidler, I.J., 1975. Biological behavior of malignant melanoma cells correlated to their survival in vivo. Cancer Res., 35: 218-224. PMID: 1109790

Filatenkov, A.A., E.L. Jacovetty, U.B. Fischer, J.M. Curtsinger and M.F. Mescher et al., 2006. CD4 $\mathrm{T}$ cell-dependent conditioning of dendritic cells to produce IL-12 results in CD8-mediated graft rejection and avoidance of tolerance. J. Immunol., 174: 6909-6917. PMID: 15905533

Fricke, I. and D.I. Gabrilovich, 2006. Dendritic cells and tumor microenvironment: A dangerous liaison. Immunol. Invest., 35: 459-483. PMID: 16916762

Garrido, F., T. Cabrera, M.A. Lopez-Nevot and F. RuizCabello, 1995. HLA class I antigens in human tumors. Adv. Cancer Res., 67: 155-195. PMID: 8571814

Geissmann, F., P. Revy, A. Regnault, Y. Lepelletier and M. Dy et al., 1999. TGF-beta 1 prevents the noncognate maturation of human dendritic Langerhans cells. J. Immunol., 162: 4567-4575. PMID: 10201996

Hahne, M., D. Rimoldi, M. Schroter, P. Romero and M. Schreier et al., 1996. Melanoma cell expression of Fas (Apo-1/CD95) ligand: Implications for tumor immune escape. Science, 274: 1363-1366. PMID: 8910274

Hargadon, K.M., C.C. Brinkman, S.L. Sheasley-O'neill, L.A. Nichols and T.N. Bullock et al., 2006. Incomplete differentiation of antigen-specific CD8 T cells in tumor-draining lymph nodes. J. Immunol., 177: 6081-6090. PMID: 7056534

Hargadon, K.M., O.A. Forrest and P.R. Reddy, 2012. Suppression of the maturation and activation of the dendritic cell line DC2.4 by melanoma-derived factors. Cell. Immunol., 272: 275-282. PMID: 22051048 
He, T., C. Tang, S. Xu, T. Moyana and J. Xiang, 2007. Interferon $\gamma$ stimulates cellular maturation of dendritic cell line DC2.4 leading to induction of efficient cytotoxic $\mathrm{T}$ cell responses and antitumor immunity. Cell. Mol. Immunol., 4: 105-111. PMID: 17484804

Helden, S.V., F.V. Leeuwen and C. Figdor, 2008. Human and murine model cell lines for dendritic cell biology evaluated. Immunol. Lett., 117: 191197. PMID: 18384885

Huang, B., P.Y. Pan, Q. Li, A.I. Sato and D.E. Levy et al., 2006. Gr- $1^{+} \mathrm{CD} 115^{+}$immature myeloid suppressor cells mediate the development of tumor-induced $\mathrm{T}$ regulatory cells and $\mathrm{T}$-cell anergy in tumor-bearing host. Cancer Res., 66: 1123-1131. PMID: 16424049

Ilkovitch, D. and D.M. Lopez, 2008. Immune modulation by melanoma-derived factors. Exp. Dermatol., 17: 977-985. PMID: 18643844

Janeway, C.A. Jr. and R. Medzhitov, 2002. Innate immune recognition. Annu. Rev. Immunol., 20: 197216. PMID: 11861602

Jiang, X., C. Shen, J. Rey-Ladino, H. Yu and R.C. Brunham, 2008. Characterization of murine dendritic cell line JAWSII and primary bone marrow-derived dendritic cells in chlamydia muridarum antigen presentation and induction of protective immunity. Infect. Immun., 76: 23922401. PMID: 18362126

Joffre, O., M.A. Nolte, R. Sporri and C. Reis e Sousa, 2009. Inflammatory signals in dendritic cell activation and the induction of adaptive immunity. Immunol. Rev., 227: 234-247. PMID: 19120488

Koneru, M., D. Schaer, N. Monu, A. Ayala and A.B. Frey, 2005. Defective proximal TCR signaling inhibits CD8+ tumor-infiltrating lymphocyte lytic function. J. Immunol., 174: 1830-1840. PMID: 15699109

Kusmartsev, S., Y. Nefedova, D. Yoder and D.I. Gabrilovich, 2004. Antigen-specific inhibition of CD8+ $\mathrm{T}$ cell response by immature myeloid cells in cancer is mediated by reactive oxygen species. J. Immunol., 172: 989-999. PMID: 14707072

Lin, A., A. Schildknecht, L.T. Nguyen and P.S. Ohashi, 2010. Dendritic cells integrate signals from the tumor microenvironment to modulate immunity and tumor growth. Immunol. Lett., 127: 77-84. PMID: 19778555

MacKay, V. and E. Moore, 1997. Immortalized dendritic cells. U.S. Patent.
Matzinger, P.,1994. Tolerance, danger and the extended family. Annu. Rev. Immunol., 12: 991-1045. PMID: 8011301

Mortarini, R., A. Piris, A. Maurichi, A. Molla and I. Bersani et al., 2003. Lack of terminally differentiated tumor-specific CD8+ T cells at tumor site in spite of antitumor immunity to self-antigens in human metastatic melanoma. Cancer Res., 63: 2535-2545. PMID: 12750277

Olson, T.S. and K. Ley, 2002. Chemokines and chemokine receptors in leukocyte trafficking. Am. J. Physiol. Regulatory Integr. Comp. Physiol., 283: R7-R28. PMID: 12069927

Ostrand-Rosenberg, S. and P. Sinha, 2009. Myeloidderived suppressor cells: Linking inflammation and cancer. J. Immunol., 182: 4499-4506. PMID: 19342621

Peter, I., A. Mezzacasa, P. LeDonne, R. Dummer and S. Hemmi, 2001. Comparative analysis of immunocritical melanoma markers in the mouse melanoma cell lines B16, K1735 and S91-M3. Melanoma Res., 11: 21-30. PMID: 11254112

Rabinovich, G., D. Gabrilovich and E. Sotomayor, 2007. Immunosuppressive Strategies that are Mediated by Tumor Cells. Annu. Rev. Immunol., 25: 267-296. PMID: 17134371

Radoja, S., M. Saio, D. Schaer, M. Koneru and S. Vukmanovic et al., 2001. CD8(+) tumor-infiltrating $\mathrm{T}$ cells are deficient in perforin-mediated cytolytic activity due to defective microtubule-organizing center mobilization and lytic granule exocytosis. J. Immunol., 167: 5042-5051. PMID:11673513

Re, F. and J.L. Strominger, 2001. Toll-like receptor 2 (TLR2) and TLR4 differentially activate human dendritic cells. J. Biol. Chem., 276: 37692-37699. PMID: 11477091

Restifo, N.P., F. Esquivel, Y. Kawakami, J.W. Yewdell and J.J. Mule et al., 1993. Identification of human cancers deficient in antigen processing. J. Exp. Med., 177: 265-272. PMID: 8426105

Rhule, A., B. Rase, J.R. Smith and D.M. Shepherd, 2008. Toll-like receptor ligand-induced activation of murine DC2.4 cells is attenuated by Panax notoginseng. J. Ethnopharmacol., 116: 179-186. PMID: 18164154

Rosenberg, S.A. and D.E. White, 1996. Vitiligo in patients with melanoma: Normal tissue antigens can be targets for cancer immunotherapy. J. Immunother. Emphasis. Tumor Immunol., 19: 8184. PMID: 8859727 
Sallusto, F., D. Lenig, C.R. Mackay and A. Lanzavecchia, 1998. Flexible programs of chemokine receptor expression on human polarized T helper 1 and 2 lymphocytes. J. Exp. Med., 187: 875-883. PMID: 9500790

Shankaran, V., H. Ikeda, A.T. Bruce, J.M. White and P.E. Swanson et al., 2001. IFNgamma and lymphocytes prevent primary tumour development and shape tumour immunogenicity. Nature, 410: 1107-1111. PMID: 11323675

Shen, Z., G. Reznikoff, G. Dranoff and K. Rock, 1997. Cloned dendritic cells can present exogenous antigens on both MHC Class I and MHC Class II Molecules. J. Immunol., 158: 2723-2730. PMID: 9058806

Sousa, C.R., 2006. Dendritic cells in a mature age. Nat. Rev. Immunol., 6: 476-483. PMID: 16691244

Terabe, M., S. Matsui, J.M. Park, M. Mamura and N. Noben-Trauth et al., 2003. Transforming growth factor-beta production and myeloid cells are an effector mechanism through which CD1d-restricted $\mathrm{T}$ cells block cytotoxic $\mathrm{T}$ lymphocyte-mediated tumor immunosurveillance: Abrogation prevents tumor recurrence. J. Exp. Med., 198: 1741-1752. PMID: 14657224
Whiteside, T.L., 2004. Down-regulation of zeta-chain expression in T cells: A biomarker of prognosis in cancer? Cancer Immunol. Immunother, 53: 865-878. PMID: 15118842

Yamaguchi, Y., H. Tsumura, M. Miwa and K. Inaba, 1997. Contrasting effects of TGF-beta 1 and TNFalpha on the development of dendritic cells from progenitors in mouse bone marrow. Stem Cells, 15: 144-153. PMID: 9090791

Yamshchikov, G., L. Thompson, W.G. Ross, H. Galavotti and W. Aquila et al., 2001. Analysis of a natural immune response against tumor antigens in a melanoma survivor: Lessons applicable to clinical trial evaluations. Clin. Cancer Res., 7: 909s-916s. PMID: 11300491

Yang, L. and D.P. Carbone, 2004. Tumor-host immune interactions and dendritic cell dysfunction. Adv. Cancer Res., 92: 13-27. PMID: 15530555

Zippelius, A., P. Batard, V. Rubio-Godoy, G. Bioley and D. Lienard et al., 2004. Effector function of human tumor-specific CD8 T cells in melanoma lesions: A state of local functional tolerance. Cancer Res., 64: 2865-2873. PMID: 15087405 